\title{
Advancement of chromosome science in the genomics era
}

\author{
Nam-Soo Kim ${ }^{1}$ (1) \\ Received: 20 January 2021 / Accepted: 26 January 2021 / Published online: 25 February 2021 \\ (c) The Genetics Society of Korea 2021
}

In 1946, an eminent plant cytogeneticist Hitoshi Kihara coined an aphorism "The history of the earth is recorded in the layers of its crust; the history of all organisms is inscribed in the chromosomes" (Crow 1994). We are all aware that chromosomes are carriers of genetic materials that pass the exact genetic information to the next generations. Chromosomes are linear structures with genes located along the specific sites of the chromosomes. However, chromosomes are stuffed with highly non-genic repetitive sequences so that only small fractions of the genomes encode proteins. Thus chromosomes are highly dynamic structures that ensure the transfer of intact genetic materials to next generations as well as regulating the expression of genes in the ocean of repetitive DNA sequences. The wealth genome sequences enhance our understanding of the chromosome dynamics. There was a conference of the 7th AsianPacific Chromosome Colloquium in November 26-28, 2020, in Pusan, Korea. The theme was "Advancement of chromosome science in the genomics era". The conference was held virtual online in both oral and poster presentation due to the COVID-19 pandemic. There were five sessions; Chromosome structure, Sex chromosomes and B-chromosomes, Chromosomes and evolution, genomics and chromosomes, and epigenomics and chromosomes. Of the 26 speakers in the symposium, ten speakers summarized their presentations and submitted their articles to this special issues. The followings are summaries of the articles.

Tada et al. (2021) demonstrated the evidence for divergence of DNA methylation maintenance and a conserved inhibitory mechanism from DNA demethylation in chickens and mammals. The role of DNA methylation is wellknown in epigenetic gene regulation which is evolutionary conserved from single cell yeasts to mammals. The levels and patterns of DNA methylation are regulated by a balance

Nam-Soo Kim

kimnamsu@kangwon.ac.kr

1 Department of Molecular Bioscience, Kangwon National University, Chuncheon 24341, Korea of antagonistic enzyme functions, DNA methyltransferases DNMT1/3A/3B and methylcytosine dioxygenases TET1/2/3. In mice, TET enzyme mediates the conversion of cytosine methylation $(5 \mathrm{mC})$ to 5 -hydroxymethylcytosine and initiation of global loss of $5 \mathrm{mC}$ at the beginning of fertilization until gastrulation stage. However, the $5 \mathrm{mC}$ level increased when the cell differentiations occur during early embryonic development. The authors checked the global loss and gain of DNA methylation whether different regulation exists in diverged species such as chicken and mammals. The results revealed that chicken and mammals shared a common chromatin-based regulation of TET-DNA success, but chicken DNMT1 was involved in different target sequence recognition systems, suggesting that factors inducing DNMT-DNA association had already diverged between egg laying bird species and placenta mammalian species.

Epigenetic regulation is a fundamental mechanism in eukaryotic gene expression. Tam and Leung (2021) reviewed current states of single-cell transcriptomic and epigenomic studies in understanding the complex pathologies of chronic inflammatory diseases (CIDs). CIDs are a diverse class of autoimmune diseases characterized by dysregulated and sustained immune responses. Approximately $60 \%$ of Americans suffer from some form of CIDs and complications relating to these diseases are significant causes of death. However, exact triggers and mechanisms underlying many CIDs still remain elusive. The authors reviewed the current understanding of an association between epigenomic and transcriptomic dysregulation and the phenotypes of CIDs, specially discussed in depth on the epigenetic changes at cis-regulatory elements (CREs). Dysregulation of transposable elements (TEs) could serve as CREs or trigger molecular mimicry in CIDs. TEs constitute about $45 \%$ of human genome and a number of reports have described the aberrant expression of retrotransposons in CIDs. For CID characterization and diagnosis, the recent advancements of sequencing technologies (i.e., single-cell genomics) have generated many useful data in understanding the etiology of these complex diseases. The authors provided a most updated summary table 
that contains published CID-studies utilizing single-cell transcriptomic and epigenomic approaches.

Shim (2021) summarized the recent advancements in super-resolution optical microscopy to localize the 3D coordinates of genomic loci in individual cells. The tightly packed chromosomes are highly dynamic structures that undergo replication, transcription and segregation. Thus, molecular and cell biologist have always been interested in the fine chromatin structures to solve the genome organization and regulation of chromosomal domains in gene expression. In her review, sequencing coupled with chromosome conformation capture (3C) method explained how to visualize the genome organization units such as $A / B$ compartments, topologically associated domains (TADs), sub-TADs and loops. She also touched the super-resolution fluorescence microscopy in visualizing the chromatin structure from $300 \mathrm{~nm}$ to $10-30 \mathrm{~nm}$ length scale. The singlemolecule localization microscopy (STORM) was introduced in detail to show the association of histone proteins for "nucleosome clutches" in 30-50 nm structure. Oligopaints combined with STORM (OligoSTORM) was demonstrated to unveil the distinct folding structures of genomic regions with different epigenetic states. Besides these microscopy techniques, she also introduced how the live-cell labelling methods are being used for imaging specific genomic loci.

Satheesh et al. (2021) presented a short review on the recent NGS techniques to detect the active transposable elements (TEs). TEs have unwelcomed monikers such as "genomic parasites" or "genomic junks". However, the growing number of species with whole genome sequenced revealed that TEs are genuine genomic treasures for shaping the current genomes by regulating gene expression, providing raw materials in evolution, and creating new genes etc. Because active TEs can often destabilize genome structure or disrupt gene expression by landing into the genes, genomes usually have machineries including methylation of the TE sequences to suppress the TE activity. However, the TE insertions can often lead to novel phenotypes such as Chardonnay variety in grapes by the LTR-retrotransposon Gretl insertion into the promoter Vvmybl gene. The authors introduced the methods to detect the active LTR-retrotransposons by high through-put genome sequencing techniques. They introduced three methods; ALE-seq (amplification of LTR of ecIDNAs followed by sequencing), Mobilome-seq, and VLP-seq (virus-like particle sequencing). They summarized and compared the three techniques for practical use in detecting the active LTR-retrotransposons in major cereal crop genomes.

Liu et al. (2021) reviewed the current understanding of the centromere with an emphasis on the roles of centromeric RNAs in centromere formation and function. Our understanding of the centromere and kinetochore has been expanded progressively with the aid of genomics and bioinformatics over the last decade. Although the functions of centromeres are evolutionarily conserved from yeasts to multicellular eukaryotes, the DNAs constituting centromeres are highly variable in sequence and evolve quickly, suggesting that there must be some epigenetic marks to establish centromere state. Because centromeres are typically formed on highly repetitive DNAs, the classical view was that transcription activity was silent in centromeres. However, low level of gene transcription occurs from the genes residing centromeres. The centromeric transcripts are variable in size but they function in a conserved epigenetic mechanism to regulate centromeres across species. The authors reviewed the roles of these cenRNA for maintaining proper function of the centromeres, for CENP-A loading onto centromere, for structuring kinetochores and therefore accurate chromosome segregation during cell division by acting on pericentromeric heterochromatin assembly for proper topological organization of centromeric chromatin via RNA:DNA hybrid. There are still a few questions remaining to be answered on the centromeres such as the recognition and targeting of the centromeric DNA by CENH3, factors involved in centromere assembly and function. They suggested that dissecting different centromeric RNA-binding proteins may give some clues on these questions.

Hayashi et al. (2021) presented an article entitled "Expression of TAS2R14 in the intestinal endocrine cells of non-human primates". Taste perception is an important trait for providing information about food. T2Rs are heterotrimeric $\mathrm{G}$ protein-coupled taste receptors expressed on the surface of the tongue to taste bitterness. Genes encoding T2Rs were diversified from a common ancestor by gene duplication and chromosomal rearrangement. The authors analyzed the expression of bitter taste receptor, TAS2R14, using RT-PCR and immunohistochemical staining methods in the rhesus macaques which is a common non-human primate model species. The results showed that mean expression levels of TAS2R14 were similar in the duodenum, ileum, and colon, but the expressions were lower than those in circumvallate papillae. The results of immunohistochemical analysis showed TAS2R14 immunoreactivity in enteroendocrine cells positive for cholecystokinin, serotonin, and the $\mathrm{G}$ protein GNAT3. Thus, the authors suggested that TAS2R14 is broadly expressed in the intestine, mainly in the enteroendocrine cells, and promotes hormone secretion in response to bitter stimuli.

Shim et al. (2021) reviewed the cytogenomic evolution of Panax species by comparative genomic analysis. Ginseng (Panax ginseng) is an important medicinal crop. However, genome sequencing of the Panax species has been lagging behind compared to other crop species due to the complex genomic, genetic and growth habits of the Panax species. Whole genome sequences are available in two species in the genus Panax, one diploid P. notoginseng $(2 n=2 x=24)$ 
and allotetraploid $P$. ginseng $(2 n=4 x=48)$. With reference to the genome of carrot (Daucus carota), the authors estimated two rounds of whole genome duplications, once in 28 million years ago (mya) in all Araliaceae family and another recently in 2.2 mya only in $P$. ginseng and $P$. quinquefolius. The genome of $P$. panax is about $3 \mathrm{Gbp}$ in which retrotransposons were predominant families of repeats. Ty3/Gypsy family retrotransposon $P g D e l l$ and $P g D e l 2$ were useful for cytogenomic analysis because $P g D e l l$ was hybridized in whole 24 pairs of chromosomes, but the PgDel2 was hybridized only in 12 pairs of chromosomes in $P$. ginseng and $P$. quinquefolius. Because the $P g D e l 2$ is hybridized on the whole set chromosomes of the diploid $P$. notoginseng, authors concluded that the $P$. gingseng and $P$. quinquefolius are allotetraploids and the allotetraploidization occurred rather recently.

Martins and Jehangir (2021) reviewed genomic insights of B chromosomes in cichlids. B chromosomes (Bs) are supernumerary chromosomes that are found in about $15 \%$ of all extant eukaryotes. Because Bs do not affect obvious phenotypic or host fitness, they were often regarded as "selfish elements". Recent genome sequencing and RNAseq analyses, however, revealed that genome structure of Bs is composed of repeated DNAs as well as protein coding genes and non-coding rRNAs. Cichlids are one of the largest vertebrate families that are distributed in Africa and South Africa. Because many species in the Cichlidae family carry B chromosomes, cichlid fishes emerged as a model for classical cytogenetics, high scale DNA, RNA and epigenetics of B chromosomes in vertebrates. The multi-omics approach with B chromosomes projected into a new era of "B-omics". The authors presented a table that compiled the cichlid species with Bs. They touched thoroughly the genomics of cichlid Bs in several species. Several high integral genes were duplicated in Bs in cichlids, which offers some putative role for survival of Bs and furthermore plays probable functions in the host genome. Moreover, the review contains some enigmas of B and sex chromosome in cichlids because several studies recognized sequences shared between B chromosomes and sex chromosomes. Bs are found in both male and female among the cichlid fishes of Lake Victoria, whereas Bs were only present in females in cichlids of Lake Malawi. Although the association between Bs and sex chromosomes is not fully understood yet, the authors hypothesized that B chromosome bias to females might favor their drive during meiosis of female fishes.

Shin et al. (2021) published a research article "Reduced fertility caused by meiotic defects and micronuclei formation during microsporogenesis in xBrassicoraphnus". Polyploidization is an important driver in plant evolution such that over $70 \%$ of the extant flowering plants are polyploids. Many domesticated crops are allopolyploids including wheats, cotton, oil seed rape etc. Karpechenko, about 100 years ago, attempted polyploidy hybrids from crosses between Raphanus sativa and Brassica oleracea to obtain Raphanus roots and Brassica leaves (Bulletin of Botany 17:305-408, 1928). Attempts were repeated recently by crossing Brassica rapa and $R$. sativus, named xBrassicoraphanus (Lee et al. 2011, 2017), in which some of the newly formed alloployploids produced stable progenies, but some showed high rates of seed abortion and pollen deformation. Meiotic analyses of these hybrids by electron microscopy and GISH (genomic in situ hybridization) revealed abnormal chromosome behaviors such as formation of univalent and inter-chromosomal bridges. The authors showed the positive correlation between meiotic defects and the formation of micronuclei which leads to the formation of unbalanced gametes and pollen sterility.

Dat et al. (2021) presented "Comparative FISH analysis of Senna tora tandem repeats which revealed insights into the chromosome dynamics in Senna". Dysploid is a phenomenon describing different basic chromosome numbers within a genus or species (Schubert and Lysak 2011). The genus Senna (family Fabaceae) comprises about 350 species. Most Senna species are diploids with $2 n=28$, but some Senna species showed dysploidy as $2 n=22 \sim 26$. The authors carried out comparative molecular cytogenetic analysis among nine Senna species with repetitive DNA probes. They demonstrated that tandem repeats were located in several chromosomal landmarks such as telomeric, subtelomeric, pericentromeric regions, and nucleolus organizing regions (NORs), and the distributions were distinct enough in use for cytotaxonomic perspective. With the results, the authors suggested that the tandem repeats might have played an important role for dysploidy in Senna species and NOR loci carrying the tandem repeats in the intergenic spacers were involved in genome reshuffling in the genus Senna, resulting in the dysploidization.

NSK is a Guest Editor for this issue.

\section{References}

Crow JF (1994) Hitoshi Kihara, Japan's pioneer geneticist. Genetics 137:891-894

Dat TT, Waminal NE, Nguyen TH, Pellerin RJ, Kim HH (2021) Comparative analysis of Senna tora tandem repeats revealed insights into the chromosome dynamics in Senna. Genes Genom 43. https ://doi.org/10.1007/s13258-021-01051-w

Hayashi M, Inaba A, Hakukawa M, Iwatsuki K, Imai H, Masuda K (2021) Expression of TAS2R14 in the intestinal endocrine cells of non-human primates. Genes Genom 43. https://doi.org/10.1007/ s13258-021-01054-7

Lee S-S, Hwang BH, Kim TY, Yang J, Han NR, Kim J, Kim HH, Belandres HR (2017) Developing stable cultivar through microspore mutagenesis in $\times$ Brassicoraphanus Koranhort, inter-generic allopolyploid between Brassica rapa and Raphanus sativus. Am J Plant Sci 8:1345-1356 
Lee SS, Lee SA, Yang J, Kim J (2011) Developing stable progenies of $\mathrm{x}$ Brassicoraphanus, an intergeneric allopolyploid between Brassica rapa and Raphanus sativus, through induced mutation using microspore culture. Theor Appl Genet 122:885-891

Liu Q, Liu Y, Shi Q, Su H, Wang C, Birchler JA, Han F (2021) Emerging roles of centromeric RNAs in centromere formation and function. Genes Genom 43. https://doi.org/10.1007/s13258-021-01041 $-\mathrm{y}$

Martin C, Jehangir M (2021) A genomic glimpse of B chromosomes in cichlids. Genes Genom 43. https://doi.org/10.1007/s13258-02101049-4

Satheesh V, Fan W, Chu J, Cho JN (2021) Recent advancement of NGS technologies to detect active transposable elements in plants. Genes Genom 43. https://doi.org/10.1007/s13258-021-01040-z

Schubert I, Lysak M (2011) Interpretation of karyotype evolution should consider chromosome structural constraints. Trends Genet 27:207-216

Shim H, Waminal NE, Kim HH, Yang TJ (2021) Dynamic evolution of Panax species. Genes Genom 43. https://doi.org/10.1007/s1325 8-021-01047-6
Shim SH (2021) Super-resolution microscopy of genome organization. Genes Genom 43. https://doi.org/10.1007/s13258-021-01044-9

Shin H, Park HR, Park JE, Yu SH, Yi G, Kim JH, Koh W, Kim HH, Lee SS, Huh JH (2021) Reduced fertility caused by meiotic and micronuclei formation during microsporogenesis in $\mathrm{xBrassiconaphus.}$ Genes Genom 43. https://doi.org/10.1007/s13258-021-01050-x

Tada M, Hayashi A, Asano Y, Kubiura-Ishimaru M, Ito T, Yoshii M, Kimura Y, Oshimura M (2021) Evidence for divergence of DA methylation maintenance and a conserved inhibitory mechanism from DNA demethylation in chicken and mammals. Genes Genom 43. https://doi.org/10.1007/s13258-021-01046-7

Tam SKM, Leung DCU (2021) Epigenomic and transcriptomic analysis of chronic inflammatory diseases. Genes Genom. 43. https:// doi.org/10.1007/s13258-021-01045-8

Publisher's Note Springer Nature remains neutral with regard to jurisdictional claims in published maps and institutional affiliations. 